\title{
Broadly tunable photon-pair generation in a suspended-core fiber
}

\author{
Jonas Hammer $\odot,{ }^{1,2, *}$ Maria V. Chekhova $\odot,{ }^{1,2}$ Daniel R. Häupl $\odot,{ }^{1,2}$ Riccardo Pennetta $\odot,{ }^{1}$ and Nicolas Y. Joly $\oplus^{2,1}$ \\ ${ }^{1}$ Max Planck Institute for the Science of Light, Staudtstraße 2, 91058 Erlangen, Germany \\ ${ }^{2}$ Physics Department, Friedrich-Alexander University, Staudtstraße 2, 91058 Erlangen, Germany
}

(Received 5 November 2019; revised manuscript received 29 December 2019; accepted 6 March 2020; published 27 March 2020)

\begin{abstract}
Nowadays fiber biphoton sources are nearly as popular as crystal-based ones. They offer a single spatial mode and easy integrability into optical networks. However, fiber sources lack the broad tunability of crystals, which do not require a tunable pump. Here, we report a broadly tunable biphoton source based on a suspended core fiber. This is achieved by introducing pressurized gas into the fibers hollow channels, changing the step index. The mechanism circumvents the need for a tunable pump laser, making this a broadly tunable fiber biphoton source with a convenient tuning mechanism, comparable to crystals. We report a continuous shift of $0.30 \mathrm{THz} / \mathrm{bar}$ of the sidebands, using up to 25 bar of argon.
\end{abstract}

DOI: 10.1103/PhysRevResearch.2.012079

Optical fibers are an ideal platform for the generation of entangled photon-pairs (biphotons) via spontaneous fourwave mixing (FWM), due to their long light-matter interaction length. In particular, solid core fibers offer high effective nonlinearity. However, they typically lack the convenient tuning mechanism present in crystal-based biphoton sources, beside the trivial but costly scheme of tuning the pump wavelength. Biphoton and twin-beam emission from crystal sources can be easily tuned over bandwidths exceeding $100 \mathrm{THz}$ via angle tuning. This property has recently been exploited as a means for absolute spectrometer calibration [1]. Biphotons tunable over $\sim 15 \mathrm{THz}$ from a crystalline (lithium niobate) whispering gallery mode resonator have been demonstrated by heating the resonator [2]. Silicon waveguides have gained popularity as sources of entangled photon states [3], and tunability might be implemented using temperature tuning [4]. In fibers, a limited amount of tuning (a few THz) has been demonstrated by stretching or heating [5]; however, these approaches are limited by fiber damage. Meanwhile, gas-filled hollow-core fibers offer broad dispersion tuning, but implementing a biphoton source in these fibers remains a challenging task, due to their low nonlinearity. Here, we combine the high nonlinearity of a solid core fiber with the convenient tuning scheme offered by gas-filled fibers, to implement a tunable source of entangled photons. This is achieved by gas-filling the channels surrounding the core of a suspended-core fiber (SCF). SCFs are a class of index-guiding microstructured fibers, where light is guided in a glass core suspended by three glass nanomembranes. SCFs have been used in a variety of

\footnotetext{
*jonas.hammer@mpl.mpg.de
}

Published by the American Physical Society under the terms of the Creative Commons Attribution 4.0 International license. Further distribution of this work must maintain attribution to the author(s) and the published article's title, journal citation, and DOI. Open access publication funded by the Max Planck Society. applications, ranging from supercontinuum generation [6] to gas absorption spectroscopy [7], or chemical sensing [8].

In FWM, two photons of an incident beam (denoted pump) are annihilated and the energy is transferred to two daughter photons (denoted signal and idler) symmetrically spaced around the pump. The signal and idler frequencies $\omega_{\mathrm{S}}, \omega_{\mathrm{I}}$ are determined by the phase-matching conditions:

$$
\begin{gathered}
\Delta \beta=2 \beta\left(\omega_{\mathrm{P}}\right)-\beta\left(\omega_{\mathrm{S}}\right)-\beta\left(\omega_{\mathrm{I}}\right)-2 \gamma P_{\text {Pump }}=0, \\
2 \omega_{\mathrm{P}}-\omega_{\mathrm{I}}-\omega_{\mathrm{S}}=0,
\end{gathered}
$$

where $\omega_{\mathrm{P}}$ is the pump frequency and $\beta$ denotes the propagation constant. $P_{\text {Pump }}$ is the peak power of the pump beam, and $\gamma$ is the effective nonlinearity of the fiber mode. The last term in Eq. (1) corresponds to a nonlinear contribution to the phase mismatch due to cross-phase and self-phase modulation terms. Here, we refer to the blueshifted sideband as signal, and the redshifted one as idler. Equation (2) ensures energy conservation and leads to a symmetric frequency detuning of the two sidebands.

If a seeding beam at either of the sideband frequencies is present, FWM is an entirely classical phenomenon, also known as parametric amplification. It is exploited in fiberoptic parametric amplifiers or oscillators $[9,10]$. If no seeding beams are launched, FWM should be described in the framework of quantum mechanics. In this case the process can be thought of as seeded by vacuum fluctuations and produces photon-pairs at the sideband frequencies. This has been used as a source of biphotons in dispersion shifted [11,12], microstructured [13,14], or polarization maintaining fibers [15]. Recently, optical fiber tapers have gained some popularity as biphoton sources [16,17]. Fiber tapers exhibit dispersion and guidance properties similar to SCF; however, the additional structure surrounding the light-guidance region makes SCF less prone to damage and environmental influences. A tunable source of bright squeezed-vacuum twin beams based on a FWM process (modulational instability) has been 
(a)

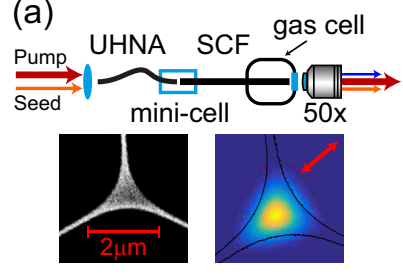

(b)

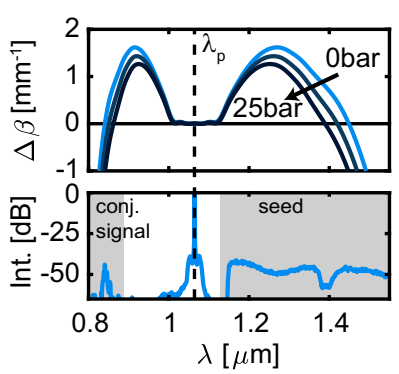

(c)

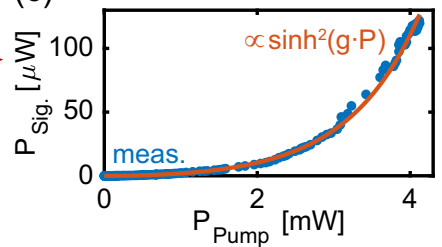

(d)

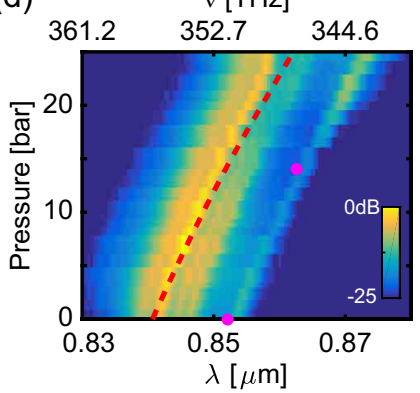

FIG. 1. Seeded FWM. (a) Setup for pressure tuning the FWM (see text). The inset shows a scanning electron micrograph of the SCF core region (left) and a numerical simulation of the fundamental mode of the fiber at $1.064 \mu \mathrm{m}$ (right; red arrow denotes the polarization). (b) Top: Simulated FWM phase mismatch for the fundamental fiber mode, for increasing pressure of argon gas filling the hollow channels of the fiber. Bottom: Experimentally measured spectrum after the SCF, when pump and seed are launched into the fiber. (c) Pump-power scaling of the signal intensity at constant seed power (blue) and its fit (orange). (d) Signal spectrum measured for increasing argon pressures. Red dashed line is an EME simulation. The two magenta circles mark the conjugate to the wavelengths marked in Fig. 3.

demonstrated in hollow-core fibers [18]. For such a source, tunability of the number of modes has been demonstrated [19] and it has been proposed for further use in a tunable biphoton source [20].

Here, we use a similar tuning principle but apply it to SCF, where the solid core provides a high nonlinearity. First, we demonstrate the continuous tuning of seeded FWM. Using parametric amplification of a broadband infrared seed, we observe a shift of $7 \mathrm{THz}$ for the emitted conjugate near-infrared sideband ( $\sim 17 \mathrm{~nm}$ at this wavelength range), when filling the fiber with up to 25 bar of argon. We then demonstrate a fiber biphoton source with very high coincidence-to-accidental ratio (CAR) and tunability over at least $4.6 \mathrm{THz}(\sim 30 \mathrm{~nm}$ measured at the red detuned wavelength, we estimate this shift can be $\sim 40 \mathrm{THz}$ if 45 bar of xenon are used as filling gas). We measured a coincidence rate of $76 \mathrm{~s}^{-1}$. Accounting for losses due to spectral filtering and fiber coupling, as well as detection efficiencies, this corresponds to a pair production rate on the order of $10^{3} \mathrm{~s}^{-1}$ at $40 \mathrm{~mW}$ continuous-wave $(\mathrm{CW})$ pump power.

Our SCF has an effective core diameter of $830 \mathrm{~nm}$, defined by a circle inscribed into the central glass region. For the parametric amplification experiment [Fig. 1(a)], the pump is a $1.064 \mu \mathrm{m}$ passively $Q$-switched laser ( $1.8 \mathrm{~ns}$ pulses, $42 \mathrm{kHz}$ repetition rate). Most of the laser power is used to generate a supercontinuum in a solid-core photonic crystal fiber [21] to serve as a seed. The supercontinuum is longpass filtered

(cut-on wavelength: $1.064 \mu \mathrm{m}$ ). Pump and seed are prepared to be copolarized and overlapping in time and space. Both beams are coupled to a single mode ultrahigh numerical aperture fiber (UHNA), which is butt-coupled to the $\sim 50-\mathrm{cm}$ long SCF inside of a mini gas cell (see $[22,23]$ for technical details). The out-coupling end of the SCF was mounted in a standard gas cell, where the pressurized gas could be introduced.

Using an eigenmode expansion (EME), based on a highresolution scanning electron micrograph of the fiber, we predict a phase-matched FWM signal for the fundamental mode near $0.84 \mu \mathrm{m}$ [Fig. 1(b), top]. The fiber presents two distinct polarization states yielding slightly different phase-matching wavelengths. We choose to work with the polarization parallel to one of the nanomembranes [inset in Fig. 1(a); red arrow marks the direction of the polarization]. The corresponding phase-matched signal at $\sim 0.84 \mu \mathrm{m}$ [Fig. 1(b), bottom] serves as a reference in order to optimize the coupling of the fundamental mode and the polarization states of the pump and seed as well as their time overlap. We also performed near-field imaging of the different wavelengths exiting the SCF, as well as polarization analysis of the fields to confirm that the process indeed only involves one fiber mode [23]. The signal intensity depends on the pump power [Fig. 1(c)] as $\sinh ^{2} G$ [24], where $G=g P_{\text {Pump }}$ is called the parametric gain. From the fit we find $g_{\text {avg }}=0.54 \mathrm{~mW}^{-1}$ in terms of the pump average power $\left(g_{\mathrm{PP}}=0.0414 \mathrm{~W}^{-1}\right.$ for peak power [23]).

Filling the SCF hollow channels with argon decreases the frequency detuning of the sidebands, as the argon pressure increases [Fig. 1(b), top]. This is because the evanescent decay length of the mode into the hollow channels scales with the wavelength. Therefore, the increasing gas refractive index (due to increasing gas pressure and therefore density) affects the propagation constant of the idler more strongly than the signal. This tuning mechanism has been used to shift the resonant third-harmonic generation wavelength in submicron tapers [25].

Increasing the pressure from 0 to 25 bar, results in a continuous shift in the signal wavelength by about $17 \mathrm{~nm}$ $(\sim 7 \mathrm{THz})$ towards smaller detuning [Fig. 1(d)]. The red dashed line corresponds to an EME simulation. The slight deviation of the simulation from the measured data is mainly due to the neglected chromatic dispersion of the filling gas, an assumption that breaks down at higher gas pressures when chromatic dispersion effects become comparable to the increase in refractive index due to increasing gas density. Fitting the experimental peak wavelength of the signal spectrum with a first-order polynomial yields an average tuning rate of $0.70 \mathrm{~nm} /$ bar $(0.30 \mathrm{THz} / \mathrm{bar})$. We note that dispersion tuning in SCF has also been demonstrated by depositing a nanofilm on the exposed core [26]; however, this mechanism is not reversible in contrast to the gas-pressure filling.

We now consider the spontaneous FWM regime, and demonstrate the effect of the gas pressure on biphoton generation. For these experiments the pump was a $1.064 \mu \mathrm{m} \mathrm{CW}$ laser and no seeding was used. The pump was launched in the same polarization state as found optimal in the previous experiments [Fig. 2(a)]. At exit from SCF, light passed through a polarizer to select the desired polarization state. The pump was suppressed by a notch filter $(-60 \mathrm{~dB})$, and the sidebands 
(a)

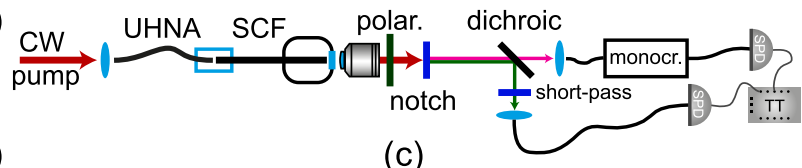

(b)

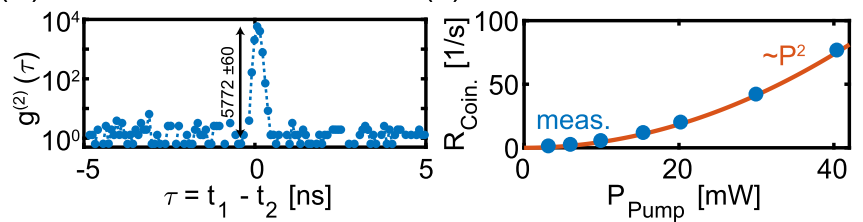

FIG. 2. Spontaneous FWM. (a) Experimental setup for measuring two-photon coincidences. A CW pump at $1.064 \mu \mathrm{m}$ is launched into the UHNA-SCF device. After recollimation, light passes through a polarizer and the pump is suppressed using a notch filter. Signal and idler photons are split on a dichroic mirror. Signal photons pass through another set of interference filters, and are coupled to a single-photon detector (SPD). Idler photons pass through a monochromator and are then sent to another SPD. Both SPDs are connected to a time tagger. (b) Measured second-order correlation function. (c) Measured coincidence rate for increasing pump powers. Orange line is a quadratic fit.

were split on a dichroic mirror. Signal photons passed through another set of interference filters (shortpass, cut-off wavelength $0.9 \mu \mathrm{m}$ ) to further suppress the pump, and were then fiber-coupled to a superconducting nanowire single-photon detector (SPD). Idler photons were directed to a fiber-coupled, home-built grating monochromator, and sent to another SPD. The passband width of the idler monochromator was $\sim 3 \mathrm{~nm}$. The SPDs were connected to a computer-controlled timetagger, registering single-photon events in either signal or idler channel, as well as coincidences between the two. We used a coincidence resolution time (bin-width) $T_{\mathrm{b}}=80 \mathrm{ps}$ for the experiments. Pair generation was characterized by measuring the normalized second-order correlation function [Fig. 2(b)], calculated as

$$
g^{(2)}(\tau)=\frac{R_{\text {coin }}(\tau)}{R_{\mathrm{S}} R_{\mathrm{I}} T_{\mathrm{b}}},
$$

where $R_{\text {coin }}(\tau)$ denotes the coincidence rate at a given time delay between two events $\tau=t_{1}-t_{2}$, and $R_{\mathrm{S}, \mathrm{I}}$ are the count rates of signal and idler detectors, respectively. The pump power was $10 \mathrm{~mW}$ coupled to the fiber, and the monochromator was set to $1.46 \mu \mathrm{m}$. The peak value of $g^{(2)}$, sometimes called CAR, is $5770 \pm 60$. Outside of the central peak, $g^{(2)}(\tau)$ drops to unity, i.e., only accidental coincidences are detected [27]. For lower pump powers we measured even higher values of the CAR exceeding $10^{4}$ [23]. In Fig. 2(c) the measured maximum coincidence rate [i.e., the value of $R_{\text {coin }}(\tau=0)$ ] is shown as a function of the coupled CW pump power. This includes detection and coupling losses after SCF. The fit in Fig. 2(c) is a parabola, corresponding to the low gain expansion of the theoretical power scaling. Through comparison with the previously found value of $g_{\mathrm{PP}}$ we can estimate the parametric gain as $G \approx 4 \times 10^{-4}$ at $10 \mathrm{~mW}$ pump power.

The major background to the biphotons stems from spontaneous Raman scattering. While the sideband detuning is larger than the typical extent of the fused silica Raman spectrum, photons from concatenated Raman processes can reach the

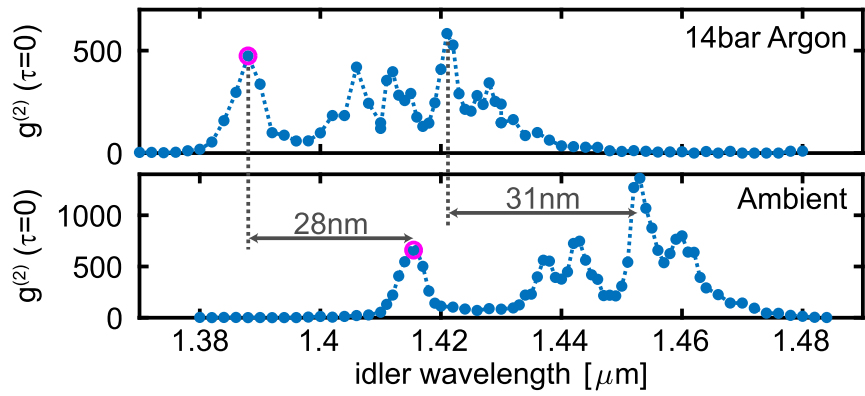

FIG. 3. Tuning of spontaneous FWM. Peak value of the secondorder correlation function measured versus the idler wavelength for SCF at ambient pressure (bottom) and filled with 14 bar argon (top). The spectral features shift by about $30 \mathrm{~nm}$. The two magenta circles are the idler wavelengths corresponding to the marked wavelengths in Fig. 1.

idler wavelength. Cooling the fiber has been demonstrated as an effective way to reduce these scattering events $[28,29]$.

To study the effect of gas-pressure tuning on the spontaneous FWM we performed a set of measurements, where the SCF was either in air (ambient pressure) or filled with 14 bar of argon. In each measurement the pump power was $20 \mathrm{~mW}$, and we scanned the monochromator across the spectral region where biphoton emission is expected. At each monochromator setting we collected data for $60 \mathrm{~s}$. The CAR versus the monochromator setting is shown in Fig. 3, for the case of air and 14 bar argon in the SCF. Similar to the seeded case, with increasing argon pressure the emission spectrum shifted to smaller detunings with the same tuning rate $(\sim 0.3 \mathrm{THz} / \mathrm{bar})$.

The $g^{(2)}$ spectrum demonstrates features very similar to those obtained in the seeded case. In particular, the two magenta markers in Fig. 3 correspond to the conjugates of the signal wavelengths that were marked in Fig. 1(c) at the same pressures. The normalized second-order correlation function measured at 14 bar is slightly reduced compared to the measurement at ambient pressure, possibly due to a higher parametric gain and higher count rates.

We verified the frequency entanglement of the photon-pairs comparing conditional and unconditional two-coincidence measurements. In Fig. 4(a) the peak value of $g^{(2)}$ is plotted as a function of the monochromator wavelength; the experimental conditions were similar to the ambient case in Fig. 3. The blue dots in Fig. 4(a) correspond to a measurement where all signal photons are directed to the SPD (unconditional measurement). The orange dots correspond to a conditional measurement, i.e., only photons of a specific wavelength $(0.85 \mu \mathrm{m})$ reach the signal SPD. The spectrum of coincidences is much narrower in this scenario, a typical sign for frequency entanglement of the photon-pairs [30,31].

Additionally we performed a stimulated emission tomography (SET) measurement. SET retrieves the joint spectral intensity (JSI) of a biphoton source by launching the pump alongside a narrow-band tunable seed to the system $[32,33]$. To match the tuning range of our seeding laser (telecom $\mathrm{O}$ band, $\sim 40 \mathrm{MHz}$ linewidth), we used a fiber with slightly smaller effective core diameter and therefore smaller frequency detuning; we denote the fiber SCF2. The fiber shows otherwise very similar properties. The SET setup is shown 
(a)

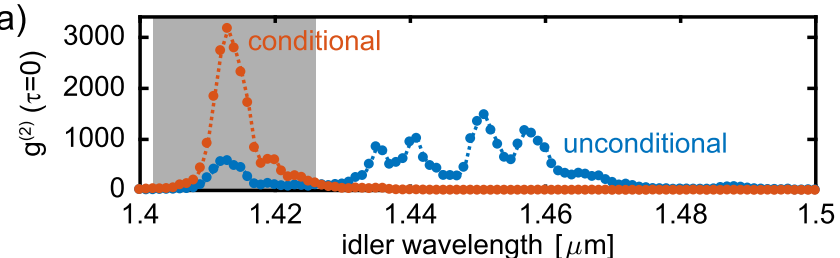

(b)

(c)
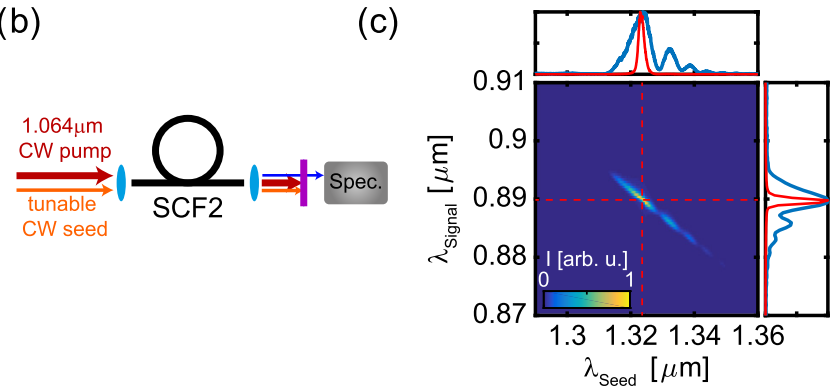

FIG. 4. Frequency entanglement. (a) Peak value of $g^{(2)}$ as a function of the monochromator wavelength measured at ambient pressure. For the unconditional measurement (blue) the signal photons are unfiltered, whereas for the conditional measurement (orange) a bandpass filter $(0.85 \mu \mathrm{m})$ is inserted in front of the signal SPD. The gray box corresponds to the conjugate of the $0.85 \mu \mathrm{m}$ bandpass full width at half maximum region. (b) Setup for a stimulated emission tomography (SET) measurement of SCF2 (different phase-matching point compared to SCF used before; length $\sim 12 \mathrm{~cm}$ ). (c) SET measurement result (heatmap). The line plots (top and right) are the marginal distributions (blue) and cuts along the dashed lines (red).

in Fig. 4(b); pump and seed (both CW and copolarized) are launched into SCF2. At exit from the fiber seed and pump are blocked and only the conjugate signal is recorded on a CCD spectrometer. The JSI is retrieved by scanning the tunable laser across the phase-matching point [Fig. 4(c)]. From the JSI we can confirm a high degree of frequency entanglement. The marginal distributions (blue line plots) and the cuts through the JSI (red line plots) correspond to unconditional and conditional measurements at the respective cross section.

We want to note that the overall shape of the emission spectra measured from our SCF are due to inhomogeneities of the fibers, which we confirmed via cutback measurements. We estimate a deviation of $\pm 2.5 \mathrm{~nm}$ of the effective core diameter over a fiber length of $\sim 50 \mathrm{~cm}$ [23]. This could be potentially improved during fiber fabrication, or by careful selection of a shorter fiber piece with less fluctuations. The overall principle of our tuning mechanism, etc, is, however, not affected by this.

In conclusion, we have demonstrated a tunable source of entangled photons, in which the spontaneous emission spectrum can be shifted by $0.70 \mathrm{~nm} /$ bar on the signal side by changing the pressure of the filling gas (argon). Our method allows tuning over a wide range of wavelengths, limited only by the maximum achievable refractive index of the filling gas. For the SCF in our experiments we estimate a sideband shift of $\sim 40 \mathrm{THz}(0.84-0.95 \mu \mathrm{m})$ when filling the fiber with up to 45 bar of xenon. In the seeded case such a system might be used to implement an all-fiber tunable parametric amplifier. The use of a solid-core, small-mode area fiber facilitates the use of low-power pump lasers, which may be of interest for small-footprint devices. Furthermore, the same principle could be used for tuning the phase matching of FWM or parametric down-conversion in other types of exposed core waveguides, e.g., on-chip ridge waveguides.

We acknowledge funding by Deutsche Forschungsgemeinschaft (DFG) (CH-1591/3-1 and JO-1090/3-1), and the Max Planck School of Photonics.
[1] S. Lemieux, E. Giese, R. Fickler, M. V. Chekhova, and R. W. Boyd, A primary radiation standard based on quantum nonlinear optics, Nat. Phys. 15, 529 (2019).

[2] M. Förtsch, J. U. Fürst, C. Wittmann, D. Strekalov, A. Aiello, M. V. Chekhova, C. Silberhorn, G. Leuchs, and C. Marquardt, A versatile source of single photons for quantum information processing, Nat. Commun. 4, 1818 (2013).

[3] L.-T. Feng, G.-C. Guo, and X.-F. Ren, Progress on integrated quantum photonic sources with silicon, Adv. Quantum Technol. 3, 1900058 (2020).

[4] X. Wang, A. Lentine, C. DeRose, A. L. Starbuck, D. Trotter, A. Pomerene, and S. Mookherjea, Wide-range and fast thermallytunable silicon photonic microring resonators using the junction field effect, Opt. Express 24, 23081 (2016).

[5] E. Ortiz-Ricardo, C. Bertoni-Ocampo, Z. Ibarra-Borja, R. Ramirez-Alarcon, D. Cruz-Delgado, H. Cruz-Ramirez, K. Garay-Palmett, and A. B. U'Ren, Spectral tunability of twophoton states generated by spontaneous four-wave mixing: Fibre tapering, temperature variation and longitudinal stress, Quantum Sci. Technol. 2, 034015 (2017).

[6] L. Fu, B. K. Thomas, and L. Dong, Efficient supercontinuum generations in silica suspended core fibers, Opt. Express 16, 19629 (2008).
[7] A. S. Webb, Suspended-core holey fiber for evanescent-field sensing, Opt. Eng. 46, 010503 (2007).

[8] A. M. Cubillas, S. Unterkofler, T. G. Euser, B. J. M. Etzold, A. C. Jones, P. J. Sadler, P. Wasserscheid, and P. St. J. Russell, Photonic crystal fibres for chemical sensing and photochemistry, Chem. Soc. Rev. 42, 8629 (2013).

[9] J. P. Pocholle, J. Raffy, M. Papuchon, and E. Desurvire, Raman and four photon mixing amplification in single mode fibers, Opt. Eng. 24, 600 (1985).

[10] J. Hansryd, P. Andrekson, M. Westlund, J. Li, and P.-O. Hedekvist, Fiber-based optical parametric amplifiers and their applications, IEEE J. Sel. Top. Quantum Electron. 8, 506 (2002).

[11] X. Li, J. Chen, P. Voss, J. Sharping, and P. Kumar, Allfiber photon-pair source for quantum communications: Improved generation of correlated photons, Opt. Express 12, 3737 (2004).

[12] H. Takesue and K. Inoue, Generation of polarization-entangled photon pairs and violation of Bell's inequality using spontaneous four-wave mixing in a fiber loop, Phys. Rev. A 70, 031802(R) (2004).

[13] J. G. Rarity, J. Fulconis, J. Duligall, W. J. Wadsworth, and P. St. J. Russell, Photonic crystal fiber source 
of correlated photon pairs, Opt. Express 13, 534 (2005).

[14] J. Fan, A. Migdall, and L. J. Wang, Efficient generation of correlated photon pairs in a microstructure fiber, Opt. Lett. 30, 3368 (2005).

[15] B. J. Smith, P. Mahou, O. Cohen, J. S. Lundeen, and I. A. Walmsley, Photon pair generation in birefringent optical fibers, Opt. Express 17, 23589 (2009).

[16] J.-H. Kim, Y. S. Ihn, Y.-H. Kim, and H. Shin, Photon-pair source working in a silicon-based detector wavelength range using tapered micro/nanofibers, Opt. Lett. 44, 447 (2019).

[17] A. A. Shukhin, J. Keloth, K. Hakuta, and A. A. Kalachev, Heralded single photon and correlated photon pair generation via spontaneous four-wave mixing in tapered optical fibers, arXiv: 1910.12918.

[18] M. A. Finger, T. S. Iskhakov, N. Y. Joly, M. V. Chekhova, and P. St. J. Russell, Raman-Free, Noble-Gas-Filled PhotonicCrystal Fiber Source for Ultrafast, Very Bright Twin-Beam Squeezed Vacuum, Phys. Rev. Lett. 115, 143602 (2015).

[19] M. A. Finger, N. Y. Joly, P. S. J. Russell, and M. V. Chekhova, Characterization and shaping of the time-frequency Schmidt mode spectrum of bright twin beams generated in gas-filled hollow-core photonic crystal fibers, Phys. Rev. A 95, 053814 (2017).

[20] M. Cordier, A. Orieux, B. Debord, F. Gérome, A. Gorse, M. Chafer, E. Diamanti, P. Delaye, F. Benabid, and I. Zaquine, Active engineering of four-wave mixing spectral correlations in multiband hollow-core fibers, Opt. Express 27, 9803 (2019).

[21] W. J. Wadsworth, N. Joly, J. C. Knight, T. A. Birks, F. Biancalana, and P. St. J. Russell, Supercontinuum and fourwave mixing with Q-switched pulses in endlessly single-mode photonic crystal fibres, Opt. Express 12, 299 (2004).

[22] R. Pennetta, S. Xie, F. Lenahan, M. Mridha, D. Novoa, and P. St. J. Russell, Fresnel-Reflection-Free Self-Aligning Nanospike Interface between a Step-Index Fiber and a Hollow-Core Photonic-Crystal-Fiber Gas Cell, Phys. Rev. Appl. 8, 014014 (2017).
[23] See Supplemental Material at http://link.aps.org/supplemental/ 10.1103/PhysRevResearch.2.012079 for estimation of the parametric gain, polarization dependence of the phase matching, origin of the shape of the emission spectrum, experimental implementation of the minicell, and measurement of high CAR.

[24] G. Agrawal, Nonlinear Fiber Optics, 5th ed. (Academic Press, New York, 2012).

[25] J. Hammer, A. Cavanna, R. Pennetta, M. V. Chekhova, P. St. J. Russell, and N. Y. Joly, Dispersion tuning in sub-micron tapers for third-harmonic and photon triplet generation, Opt. Lett. 43, 2320 (2018).

[26] S. C. Warren-Smith, M. Chemnitz, H. Schneidewind, R. Kostecki, H. Ebendorff-Heidepriem, T. M. Monro, and M. A. Schmidt, Nanofilm-induced spectral tuning of third harmonic generation, Opt. Lett. 42, 1812 (2017).

[27] The seemingly high fluctuations in this region are due to the low count rates for these time bins.

[28] S. D. Dyer, M. J. Stevens, B. Baek, and S. W. Nam, Highefficiency, ultra low-noise all-fiber photon-pair source, Opt. Express 16, 9966 (2008).

[29] S. D. Dyer, B. Baek, and S. W. Nam, High-brightness, lownoise, all-fiber photon pair source, Opt. Express 17, 10290 (2009).

[30] Y. M. Mikhailova, P. A. Volkov, and M. V. Fedorov, Biphoton wave packets in parametric down-conversion: Spectral and temporal structure and degree of entanglement, Phys. Rev. A 78, 062327 (2008).

[31] G. Brida, V. Caricato, M. V. Fedorov, M. Genovese, M. Gramegna, and S. P. Kulik, Characterization of spectral entanglement of spontaneous parametric-down conversion biphotons in femtosecond pulsed regime, Europhys. Lett. 87, 64003 (2009).

[32] M. Liscidini and J. E. Sipe, Stimulated Emission Tomography, Phys. Rev. Lett. 111, 193602 (2013).

[33] B. Fang, O. Cohen, M. Liscidini, J. E. Sipe, and V. O. Lorenz, Fast and highly resolved capture of the joint spectral density of photon pairs, Optica 1, 281 (2014). 